\title{
Severe rhabdomyolysis associated with catha edulis(khat) use
}

\author{
Mark Tratenberg, Shikha Mehta, George Kleinman, Victor Gazivoda, I oannis Tassiulas \\ Department of Rheumatology, New York Medical College, Valhalla, NY, United States \\ Correspondence: Mark Tratenberg. Address: Department of Rhuematology, New York Medical College, Valhalla, NY, \\ United States. E-mail: Mtraiin@gmail.com
}

Received: August 5, 2014

DOI : $10.5430 /$ crcp.v2n1p87

\begin{abstract}
Severe rhabdomyolysis is a rare complication of exposure to the recreational drug khat. Adverse reactions to khat that have been reported in the literature include myocardial infarction and reversible vasoconstriction syndrome. The active ingredient, cathinone, is a naturally occurring beta-ketone amphetamine that may be responsible for this severe catecholaminergic response. Here we report a case of severe rhabdomyolysis following exposure to khat.
\end{abstract}

\section{Key words}

Khat, Rhabodmyolysis, Amphetamines, Cathinone

\section{I ntroduction}

Khat, also known as "Bath Salts", is one of the most popularly abused substances in the Middle East and East Africa. It is so widely used that is estimated that $80 \%$ of men and women chew khat in Yemen with $40 \%$ of each household annual income spent on its purchase ${ }^{[1]}$. Khat is a flowering plant (Catha edulis) native to the Horn of Africa and the Arabian Peninsula, which causes amphetamine-like sympathomimetic effects including tachycardia, hypertension as well as increased alertness and euphoria ${ }^{[2]}$. The active ingredient, cathinone ([S]-2-amino-1-phenyl-1-propanone) is a naturally occurring beta-ketone amphetamine analogue that increases the levels of dopamine in the brain by acting in the catecholaminergic synapses ${ }^{[2]}$. Although commonly used for its stimulant and euphoric properties, the chronic consumption khat leaves has been attributed to staining of the teeth and psychotic symptoms.

In the literature, khat has recently been associated with cases of myocardial infarction, strokes and reversible vasoconstriction syndrome ${ }^{[3,4]}$. Although there are case reports of rhabdomyolysis induced by intravenous synthetic cathinone ${ }^{[5]}$, we believe this is the first case of khat-induced rhabdomyolysis.

\section{Case presentation}

In November, 2013, a 51 year-old man presented to our hospital complaining of sub-acute onset of bilateral proximal lower extremity weakness, fatigue and dysphagia. Two weeks prior to admission, he had been visiting family in Yemen where he had been chewing 2-3 grams of fresh khat leaves per day for the past 14 days. He denied any other drug use or 
excess caffeine consumption. On physical examination, there were no obvious signs of trauma or skin rashes. The proximal lower extremities were non-tender on palpation and there were no joint effusions or synovitis. His Medical Research Council (MRC) Scale for muscle strength was 2/5 in the proximal lower extremity, and 5/5 in the distal lower extremity, proximal upper extremity, and distal upper extremity. Laboratory investigations showed normochromic, normocytic anemia, an elevated erythrocyte sedimentation rate of $70 \mathrm{~mm} / 1 \mathrm{hr}$, creatine kinase of $>42,000 \mathrm{IU} / \mathrm{ml}$, normal troponin, BUN $50 \mathrm{mg} / \mathrm{dl}$, creatinine $2.5 \mathrm{mg} / \mathrm{dl}$, normal uric acid, no urine eosinophils, normal serum electrolytes, elevated urine myoglobin $3.9 \mathrm{ug} / \mathrm{L}$, negative antinuclear antibodies (ANAs) autoantibodies, normal C3 and C4 complement levels and negative HIV, HCV antibodies and HBsAg in the serum. A myositis panel was negative for PL-7, PL-12, Mi-2, Ku, EJ, OJ, SRP, and Jo-1 autoantibodies. There were pigmented urinary casts in the urine but no RBC or WBC casts. Absence of chest pain and a normal electrocardiogram ruled out cardiac involvement. Renal ultrasonography did not reveal existing renal disease or obstruction of the urinary collecting system. Electromyography and nerve conduction studies were inconclusive due to his inability to contract his muscles. MRI of the lower extremities showed symmetric increased signal intensity in T1 and STIR images predominantly involving the iliopsoas, adductor, sartorius, vastus medialis, intermedius, gluteus and oblique abdominal muscles (see Figure 1). A biopsy of the left vastus medialis muscle was performed which showed an active necrotizing myopathy with mild inflammatory infiltrate compatible with a toxin exposure (see Figure 2). The biopsy specimen was negative for cytochrome oxidase deficiency (see Figure 3), a condition that non-specifically predisposes to toxic insults, and MHC class I expression, which is up-regulated in inflammatory myositis. The patient received supportive therapy with adequate intravenous fluid hydration and avoidance of khat consumption. Marked clinical improvement in lower extremity weakness, normalization of muscle enzymes, and resolution of acute renal failure occurred over the following two weeks. MRC scores of 5/5 were achieved in all extremities. He was discharged to a rehabilitation facility and on last follow up in January 2013 he was found to have elevated muscle enzyme levels after re-exposure to khat.

Figure 1. There is increased T2 signal within the musculature diffusely predominantly involving the iliopsoas musculature, adductor, sartorius, vastus medialis and intermedius bilaterally. The hamstring and gluteus musculature is also involved to a lesser extent. Increased T2 signal is also noted within the abdominal oblique musculature bilaterally.

Figure 2. Skeletal muscle biopsy (200× original magnification). Right vastus medialis biopsy shows acutely necrotic fibers (White arrows). Only rare perivascular lymphocytes are identifyed, and neither plasma cells nor lymphocytes within the muscle fibers are seen.
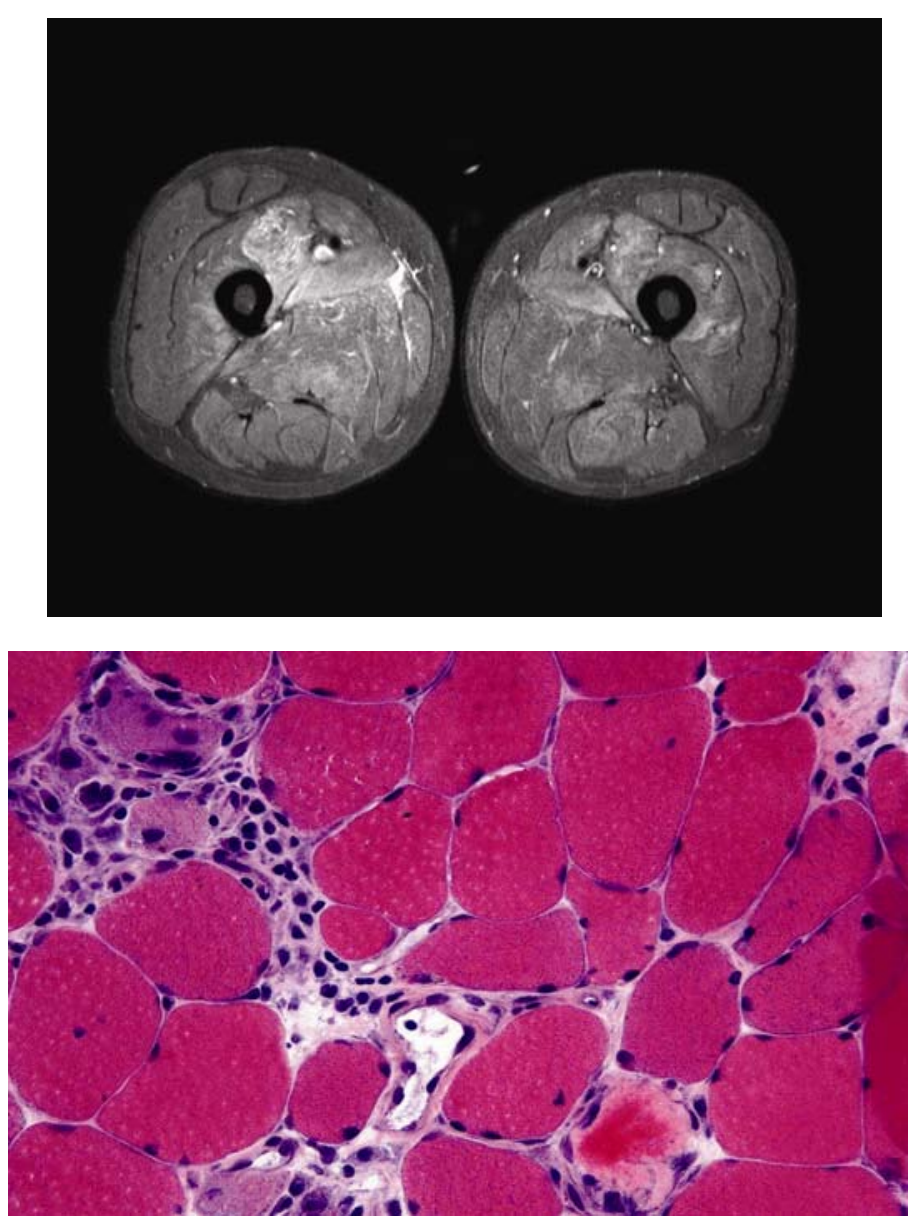

ISSN 2331-2726 E-ISSN 2331-2734 
Figure 3. Histochemical stain for cytochrome oxidase (40× magnification). There is no evidence of perifasciular atrophy.

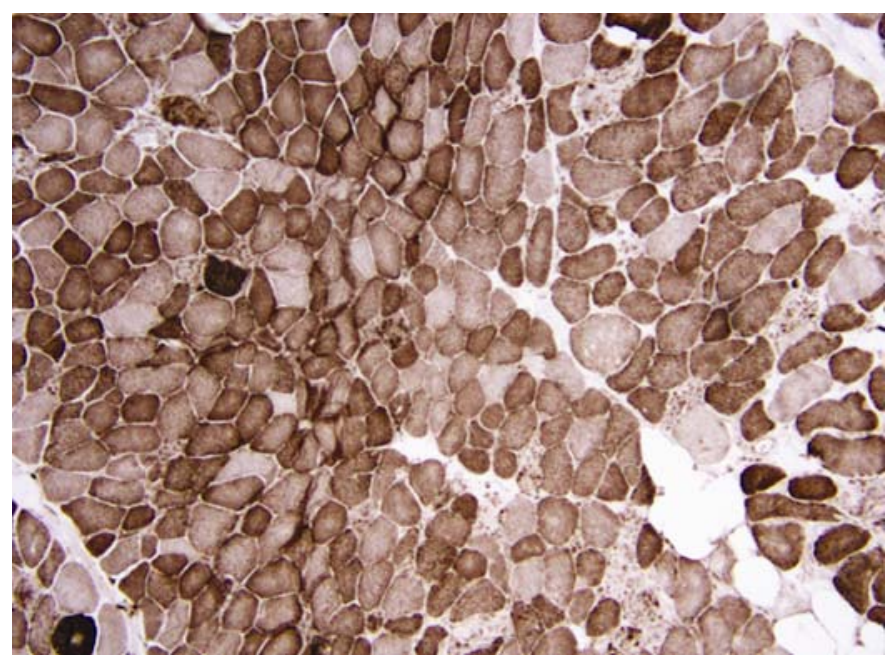

\section{Discussion}

According to the National Institute of Drug Abuse, an estimated 10 million people worldwide chew khat. Although the WHO Expert Committee on Drug Dependence (ECDD) considered khat a schedule III drug in its 1971 convention, it was not until 2002 that the WHO determined there was sufficient information to reschedule khat as a class I drug. In the United States, khat use is currently illegal in just 27 states. Increased immigration from countries such as Ethiopia, Yemen and Somalia have led to increased hospitalizations for seizures, psychosis and acute hepatitis from chewing khat.

The diagnosis of khat-induced rhabdomyolysis is difficult due to the rare incidence of disease and inability to test for a commercially available khat metabolite in the United States. Perhaps the most important issue is that most physicians in the western hemisphere may not be aware of khat and patients may not voluntarily admit to khat use as they do not consider it a drug. Imaging for muscle toxicity is non-specific and histological samples can only be used to rule out other causes of myositis and would not reveal khat toxicity as an underlying etiology. The major clue to diagnosis would be resolution of symptoms following cessation of khat exposure and recurrence of symptoms after reexposure. Other potential causes of muscle toxicity including drug interactions, metabolic causes, infections, autoimmune diseases, excess caffeine and alcohol consumption must be excluded.

We postulate that the cause of muscle toxicity may be similar to the mechanism, which has been implicated in khatinduced hepatic necrosis ${ }^{[5]}$. Khat chewing has been shown to up-regulate the pro-apoptotic protein Bax, activate caspase-8 and caspase-9 and down-regulate the expression of the anti-apoptotic protein Bcl-2 in hepatocytes ${ }^{[5]}$. Khat also triggers the generation of intracellular reactive oxygen species (ROS) and sequentially induces the sustainable activation of the c-Jun NH2-terminal kinase (JNK) pathway promoting cell apoptosis ${ }^{[5]}$.

Literature review showed that this was the first case of rhabdomyolysis secondary to khat use. As khat use is becoming more prevalent outside the Middle East, physicians should be aware of the potential side effects of this drug.

\section{Conflict of interests}

The authors declare that there is no conflict of interest.

\section{Author contribution and acknowledgement}

Dr. Mark Tratenberg wrote the manuscript. Dr. Kleinman is responsible for the pathology diagnosis. Dr. Ioannis Tassiulas, Dr, Shikha Mehta, and Dr. Victor Gazivoda are responsible for editing of the manuscript. 


\section{References}

[1] Abid MD, Chen J, Xiang M, Zhou J, Chen X, Gong F. Khat (Catha edulis) generates reactive oxygen species and promotes hepatic cell apoptosis via MAPK activation. Int J Mol Med. 2013; 32: 389-395. PMid: 23708648.

[2] Al-Motarreb A, Baker K, Broadley KJ. Khat: pharmacological and medical aspects and its social use in Yemen. Phytother Res. 2002; 16: 403-413. PMid: 12203257. http://dx.doi.org/10.1002/ptr.1106

[3] Al-Motarreb A, Shabana A, El-Menyar A. Epicardial coronary arteries in khat chewers presenting with myocardial infarction. Int J Vasc Med. 2013; 857019. PMid: 24222853.

[4] Tuladhar AM, Boogaarts HD, de Leeuw FE, van Dijk E. Reversible cerebral vasoconstriction syndrome after chewing khat leaves. Cerebrovasc Dis. 2013; 36: 158-159. PMid: 24029701. http://dx.doi.org/10.1159/000353664

[5] Patel NB. Mechanism of action of cathinone: the active ingredient of khat (Catha edulis). East Afr Med J. 2000 ; 77: $329-332$. PMid: 12858935. 